\title{
Publisher's Note: Quantum computation with realistic magic-state factories [Phys. Rev. A 95, 032338 (2017)]
}

\author{
Joe O’Gorman and Earl T. Campbell
}

(Received 14 February 2018; published 21 February 2018)

DOI: 10.1103/PhysRevA.97.029905

This paper was published online on 31 March 2017 with an error in the text on page 9. On page 9, in Sec. VIC, right-hand column, the sixteenth line of the second paragraph should read as "surface code magic-state factory of 6.3 million "data qubits" if the infidelity of operations on physical qubits is $10^{-4}$. This is based on the cost of $d^{2}$ physical qubits to store the information in the rotated lattice surface code [29]. However, for many architectures this number must be doubled to provide ancillas responsible for syndrome extraction. In this case the physical qubit overhead would be $\sim 13$ million." The text has been corrected as of 7 February 2018. The text is incorrect in the printed version of the journal. 